\title{
Getting Older Women Pregnant: Contemporary Thoughts
}

\author{
Gautam N. Allahbadia ${ }^{1}$
}

Published online: 13 November 2015

(C) Federation of Obstetric \& Gynecological Societies of India 2015

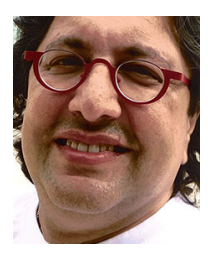

\begin{abstract}
About the Author
Gautam N Allahbadia, MD is the Editor-in-Chief of the Journal of Obstetrics \& Gynecology of India as well as the IVF Lite (Journal of Minimal Stimulation IVF). He is the Medical Director of Rotunda-The Center for Human Reproduction, the world-renowned infertility clinic at Bandra, and Rotunda-Blue Fertility Clinic and Keyhole Surgery Center, Mumbai, India. $\mathrm{He}$ is a noted world authority on ultrasound-guided embryo transfers and one of the pioneers in Third Party Reproduction in Southeast Asia. Dr. Allahbadia was responsible for India's first trans-ethnic surrogate pregnancy involving a Chinese couple's baby delivered by an unrelated Indian surrogate mother. He cherishes over 125 peer reviewed publications, 134 book chapters and 22 textbooks, the latest being a comprehensive text, entitled "Minimal Stimulation IVF", and is on the Editorial Board of several International Journals. He just completed a two year tenure as Medical Director at the New Hope IVF Clinic, Sharjah, UAE, where he introduced IVF Lite to the country. Dr. Allahbadia has recently been elected as the Vice President of the World Association of Reproductive Medicine (WARM), headquartered in Rome, and "Mumbai's Top Doc" for 2012 by a peer nomination
\end{abstract} process. You can read more about his work at www.gautamallahbadia.com.

\begin{abstract}
For women of advanced age with abnormally increased FSH levels, standardized hormonal stimulation often represents a cost-intensive procedure with a low success rate. It is well established now that with mild ovarian stimulation, there is a greater percentage of good-
\end{abstract}

Gautam N Allahbadia is the Editor-in-Chief of the Journal of Obstetrics \& Gynecology of India as well as the IVF Lite (Journal of Minimal Stimulation IVF) and Medical Director of Rotunda-The Center for Human Reproduction, Bandra, and Rotunda-Blue Fertility Clinic and Keyhole Surgery Center, Parel, Mumbai, India.

Gautam N. Allahbadia

ivfwaladoc@gmail.com

1 Rotunda-The Center for Human Reproduction, Mumbai, India quality eggs (although a smaller number) than with higherdose conventional stimulation. Mild stimulation protocols reduce the mean number of days of stimulation, the total amount of gonadotropins used and the mean number of oocytes retrieved. The proportion of high-quality and euploid embryos seems to be higher compared with conventional stimulation protocols, and the pregnancy rate per embryo transfer is comparable. Moreover, the reduced costs, the better tolerability for patients and the less time needed to complete an IVF cycle make mild approaches clinically and cost-effective over a given period of time. The low number of embryos available for transfer poses a great challenge in the management of older women going in for IVF. A potential management of these older women is to create a sufficient pool of embryos by accumulating 
vitrified good-grade embryos over several minimal stimulation and natural cycles. At the end of the accumulation process, these embryos can be subjected to a preimplantation genetic screening using next-generation sequencing and then the pool would have only chromosomal normal embryos with maximal chances of implantation. This would potentially make the chances of success for older women similar to normal responders. This management, however, is unthinkable without an outstanding vitrification program. The option of accumulating embryos has become a promising reality with the advent of vitrification technologies.

Keywords AMH · Older patients · IVF · IUI ·

Vitrification · NGS · Trophectoderm biopsy · PGS ·

Preimplantation genetic screening · PGD .

Preimplantation genetic diagnosis · IVF lite .

Minimal stimulation IVF

\section{Introduction}

Iglesias et al. [1] investigated differences in ovarian reserve markers [antimüllerian hormone (AMH) and antral follicle count (AFC)] in Indian and Spanish women. The mean age of women undergoing their first or second IVF cycle was significantly higher in Spanish than in Indian women ( $37.5 \pm 3.3$ vs. $31.5 \pm 3.8$ years). Despite this 6-year age gap, AFCs were similar $(9.5 \pm 4.7$ vs. $9.9 \pm 4.6)$, as were day-3 FSH levels $(7.5 \pm 4.5$ vs. $6.9 \pm 2.3 \mathrm{IU} / \mathrm{L})$. AMH levels were slightly lower in Spanish women $(1.6 \pm 1.7$ vs. $2.5 \pm 1.6 \mathrm{ng} / \mathrm{mL}$ ). Multivariate regression analysis showed that being Indian decreased AFC by 2.3, such that AFC in Indian women was similar to that in Spanish women 6.3 years older (95\% CI 3.39-1.10). Similar ovarian reserve markers and ovarian response were observed in women with a 6-year age difference in favor of the Spanish, suggesting ethnic differences in ovarian aging. Further research is needed to understand whether these differences are genetically induced or are caused by other variables, such as nutrition [1]. The number of women attempting to conceive between the ages of 36 and 44 has increased significantly in the last decade. While it is well established that women's reproductive success dramatically declines with age, the underlying physiological changes responsible for this phenomenon are not well understood. With assisted reproductive technologies, it is clear that oocyte quality is a likely cause since women over 40 undergoing in vitro fertilization (IVF) with oocytes donated by younger women have success rates comparable to young patients. Till a few years ago, apart from oocyte donation, there was no known intervention to improve the pregnancy outcome of older patients. Today, with the widespread use of the IVF Lite protocol [2-4] and the advent of next-generation sequencing (NGS) technology to screen embryos [5, 6], we have pregnancy rates that have almost doubled in older women from a decade back.

Goldman et al. [7] set up a randomized clinical trial to determine the optimal infertility therapy for women at the end of their reproductive potential. The study included couples with $\geq 6$ months of unexplained infertility; female partner aged 38-42 years, who were randomized to treatment with two cycles of clomiphene citrate (CC) and intrauterine insemination (IUI), follicle stimulating hormone (FSH)/IUI or immediate IVF, followed by IVF if not pregnant. They randomized 154 couples to receive CC/IUI $(N=51), \mathrm{FSH} / \mathrm{IUI}(N=52)$ or immediate IVF $(N=51)$; $140(90.9 \%)$ couples initiated treatment. The cumulative clinical pregnancy rates per couple after the first two cycles of CC/IUI, FSH/IUI or immediate IVF were 21.6, 17.3 and $49.0 \%$, respectively. After all treatments, $110(71.4 \%)$ of 154 couples had conceived a clinically recognized pregnancy and $46.1 \%$ had delivered at least one live-born baby; $84.2 \%$ of all live-born infants resulting from treatment were achieved via IVF. There were $36 \%$ fewer treatment cycles in the IVF arm compared with either $\mathrm{COH} / \mathrm{IUI}$ arm, and the couples conceived leading to a live birth after fewer treatment cycles. This randomized controlled trial in older women with unexplained infertility to compare treatment initiated with two cycles of controlled ovarian hyperstimulation/IUI versus immediate IVF demonstrated superior pregnancy rates with fewer treatment cycles in the immediate IVF 7). Success rates for women aged 40 or over with clomiphene, IUI, IUI with FSH are all extremely low, at $<1 \%$ live birth per cycle. However, IVF offers a success rate of around $13.7 \%$ per cycle. Attempting treatments other than IVF will delay conception unnecessarily [8].

In many IVF centers, it is a common practice to consider the day-3 FSH value as an end marker for the patient selection procedure. Most centers hold a day-3 FSH value of 10-15 IU/L as the upper limit to decide whether to provide IVF treatment or not, since one of the large-scale studies evaluated a day- $3 \mathrm{FSH}>15 \mathrm{IU} / \mathrm{L}$ and demonstrated a decrease in pregnancy rate [9]. Many patients having high day-3 FSH are denied IVF treatment quoting reduced ovarian reserve and low success rate and pushed toward donor egg IVF. The high base-line FSH concentration that is usually observed with older women due to lower ovarian reserve simply indicates the need for an IVF Lite protocol with no down-regulation and banking of embryos with back-to-back mild stimulation alternating with natural IVF cycles. In Zhang's series, patients were not denied treatment based on their day-3 FSH value or ovarian reserve [10]. Yet very acceptable pregnancy rates were achieved $(20 \%$ for fresh 
embryo transfers (ETs) and $41 \%$ for cryopreserved ETs). These results strengthen the argument for an IVF Lite protocol $[2-4,11,12]$ as an alternative to standard conventional IVF stimulation protocols.

\section{Discussion}

In the era before vitrification, Weghofer et al. [13] reported on 84 IVF cycles using minimal ovarian stimulation and 85 cycles with a standard long-stimulation protocol in women aged 40 and above who had slightly increased FSH levels. Minimal stimulation cycles resulted in a clinical pregnancy rate of $8.2 \%$ per started cycle and $10 \%$ per ET, whereas the control group yielded a clinical pregnancy rate of $10.6 \%$ per started cycle and of $10.7 \%$ per ET (not statistically significant). In women aged 40 and above with abnormal FSH levels, minimal stimulation protocol achieves similar pregnancy rates to a standard protocol, and thus represents a cost-effective alternative [13].

A retrospective, cohort study was performed in private infertility center to evaluate the embryological and clinical results of a large exclusive SET program according to patient age (lower or equal 29, 30-34, 35-39, 40-44 and equal to or higher than 45 years) [14]. A total of 7,244 infertile patients underwent 20,244 cycles with a clomiphene-based minimal stimulation or natural-cycle IVF protocol during 2008. Following oocyte retrieval, fertilization and embryo culture, a total of 10,401 fresh or frozen single-ET procedures were performed involving cleavagestage embryos or blastocysts. Successful oocyte retrieval rate $(78.0 \%)$ showed no age-dependent decrease until 45 years. Fertilization $(80.3 \%)$ and cleavage $(91.1 \%)$ rates were not significantly different between age groups. Blastocyst formation (70.1-22.8 \%) and overall live birth rates (LBR) (35.9-2 \%) showed an age-dependent decrease. Vitrified frozen-thawed blastocyst transfer (BT) cycles gave the highest chance of live birth per ET (41.3-6.1\%). High fertilization and cleavage rates were obtained regardless of age, whereas blastocyst formation and LBR showed an age-dependent decrease. An elective single-ET program based on a minimal ovarian stimulation protocol yielded acceptable LBR per ET in infertile patients up until their mid-40s [14]. However, in very advanced age patients (equal to or higher than 45 years old) success rates fall below $1 \%$.

IVF-ET with intracytoplasmic sperm injection (ICSI) was performed for a 45-year-old woman with a peak serum FSH level of $29 \mathrm{mIU} / \mathrm{mL}$ and a history of failing to conceive in five previous IVF-ET cycles at a younger age. A minimal FSH stimulation protocol was used [15]. A fresh transfer of a seven-cell embryo was performed on day 3. A successful pregnancy and delivery ensued. This case report establishes a precedent that a successful pregnancy following IVF-ET is possible in a woman whose serum FSH is $>15 \mathrm{mIU} / \mathrm{mL}$ and age is 45 . Of course, there is no implication that accomplishing this again in another woman with similar circumstances would be likely [15].

Zhu et al. [16] compared the clinical outcome of fresh versus vitrified-warmed BT cycles. In 110 fresh BT cycles versus 136 vitrified-warmed BT cycles performed from January 2007 to March 2010, the IR and CPR of vitrifiedwarmed BT cycles were 37.0 and $55.1 \%$, respectively, which were statistically significantly higher than the corresponding values of 25.2 and $36.4 \%$ obtained for fresh BT cycles. Vitrified-warmed BT cycles resulted in statistically significantly higher CPR and IR compared with fresh BT cycles. A new ET strategy was therefore proposed whereby fresh BT would be avoided in the initial ovarian stimulation cycle. Instead, all the patient's available blastocysts would be vitrified-warmed and transferred in subsequent cycles [16]. Kalampokas et al. [17] did a systematic review to determine whether IVF, frozen replacement cycles offer better outcomes than fresh cycles in order to support, or not, a possible shift toward total replacement of fresh IVF/ICSI cycles from frozen elective transfers (FETs). Initial results seem to support a shift in current practice toward frozen cycles. Initial results may support replacement of all fresh IVF/ICSI cycles with FETs, as this could be a safer and equally effective strategy [17]. Also, vitrification of mature oocytes and embryos obtained better clinical outcomes and did not increase the risks of DNA damage, spindle configuration, embryonic aneuploidy, and genomic imprinting as compared to fresh and slow-freezing procedures, respectively [18]. This is the global trend now, and especially for older women, poor responders and now even for hyper-responders, a deferred or remote ET is preferred [3, 4, 11, 12].

Zhang et al. [19] performed a randomized non-inferiority controlled trial with a prespecified border of $-10 \%$ comparing one cycle of mini-IVF with single ET to one cycle of conventional IVF with double ET. Five hundred and sixty-four couples were randomly assigned between February 2009 and August 2013 with 285 allocated to mini-IVF and 279 to conventional IVF. The cumulative live birth rate was $49 \%$ (140/285) for mini-IVF and $63 \%$ (176/279) for conventional IVF (RR 0.76, $95 \%$ CI 0.64-0.89). There were no cases of OHSS after mini-IVF compared to $16(5.7 \%)$ moderate/severe OHSS cases after conventional IVF. The multiple pregnancy rates were $6.4 \%$ in mini-IVF compared to $32 \%$ in conventional IVF (RR $0.25,95 \%$ CI 0.14-0.46). Gonadotropin consumption was significantly lower with mini-IVF compared to conventional IVF $(459 \pm 131$ vs. $2079 \pm 389 \mathrm{IU}$, $p<0.0001)$. Compared to conventional IVF with double ET, mini-IVF with single ET lowers live birth rate, 
completely eliminates OHSS, reduces multiple pregnancy rates and reduces gonadotropin consumption [19].

A 3-year, retrospective, single-center cohort study was conducted in a private infertility center to determine cumulative LBR per scheduled oocyte retrieval following minimal ovarian stimulation/natural-cycle IVF in unselected infertile patients [20]. A total of 727 consecutive infertile patients were analyzed who underwent 2,876 (median 4) cycles. Natural-cycle IVF or clomiphene-based minimal ovarian stimulation was coupled with single ET and increased use of delayed vitrified-warmed BT. Crude cumulative LBR were $65,60,39,15$ and $5 \%$ in patients aged 26-34, 35-37, 38-40, 41-42 and 43-44 years, respectively. Acceptable cumulative LBR are reached with an exclusive minimal ovarian stimulation/single-ET policy especially in patients aged $<38$ years but also in intermediate aged patients (38-40 years) [20].

\section{Recent Advances}

Mersereau et al. [5] compared the strategy of traditional IVF with prenatal diagnosis versus IVF with preimplantation genetic screening (IVF/PGS) to prevent aneuploid births in women with advanced maternal age. A decision tree analytic model was created to compare IVF alone versus IVF/ PGS to evaluate which strategy is the least costly per healthy (euploid) infant. Using base-case estimates of costs and probabilities in women aged 38-40 years, after a maximum of two fresh IVF cycles and two frozen cycles, the chance of having a healthy infant was $37.8 \%$ with IVF alone versus $21.7 \%$ with IVF/PGS. The average cost for each strategy is $\$ 25,700$, but the cost per healthy infant is substantially higher when IVF/PGS is applied as opposed to IVF alone ( $\$ 118,713$ vs. $\$ 68,026)$. To assess the robustness of the model, all probabilities were varied simultaneously in a Monte Carlo simulation, and in $96.2 \%$ of trials, IVF alone proved to be the most cost-effective option. Conversely, our data demonstrate that in women aged $>40$, IVF and IVF/ PGS are essentially equal in terms of cost-effectiveness $(\$ 122,000$ vs. $\$ 118,713)$ [5].

\section{Conclusions}

Different strategies of investigation and management are proposed to patients over 40 in order to overcome their infertility and improve the live birth rate in these patients. Intrauterine insemination (IUI) in women over 40 is associated with a low rate of ongoing pregnancy, and IUI should not therefore be offered always as the first line of treatment. As best demonstrated by the 2004 US National Summary and Fertility Clinic Report, which for the first time reported pregnancies and births above age 45 year, IVF in women of advanced reproductive age represents a cutting edge area of interest for improving current IVF outcomes. Access to IVF should, therefore, not be withheld based on female age and/or baseline FSH levels [21]. Furthermore, evidence has shown that, by these avantgarde techniques (minimal stimulation, trophectoderm biopsy, vitrification and NGS), older infertile women with the help of eSET may have an opportunity to increase the success of their LBR approaching those reported in younger infertility patients [6].

\section{References}

1. Iglesias C, Banker M, Mahajan N, et al. Ethnicity as a determinant of ovarian reserve: differences in ovarian aging between Spanish and Indian women. Fertil Steril. 2014;102(1):244-9. doi: 10.1016/j.fertnstert.2014.03.050.

2. Gandhi G, Allahbadia G, Kagalwala S, et al. ACCU-VIT: a new strategy for managing poor responders. Hum Reprod. 2013;28:1149-206.

3. Allahbadia GN. IVF Lite: is this the future of assisted reproduction? J Obstet Gynecol India. 2013;63:1-4.

4. Allahbadia GN. Clomiphene citrate for in vitro fertilization: the wheel turns a full circle. IVF Lite. 2015;2:47-52 (cited 28 Sep 2015).

5. Mersereau JE, Plunkett BA, Cedars MI. Preimplantation genetic screening in older women: a cost-effectiveness analysis. Fertil Steril. 2008;90(3):592-8 (Epub 14 Nov 2007).

6. Wu MY, Chao KH, Chen CD, et al. Current status of comprehensive chromosome screening for elective single-embryo transfer. Obstet Gynecol Int. 2014;2014:581783. doi: $10.1155 / 2014 / 581783$

7. Goldman MB, Thornton KL, Ryley D, et al. A randomized clinical trial to determine optimal infertility treatment in older couples: the forty and over treatment trial (FORT-T). Fertil Steril. 2014;101(6):1574-81.e1-2. doi:10.1016/j.fertnstert.2014.03.012.

8. Armstrong S, Akande V. What is the best treatment option for infertile women aged 40 and over? J Assist Reprod Genet. 2013;30(5):667-71. doi:10.1007/s10815-013-9980-6.

9. Scott RT, Toner JP, Muasher SJ. Follicle stimulating hormone levels on cycle day 3 are predictive of in vitro fertilization outcome. Fertil Steril. 1989;51:651-4.

10. Zhang J, Chang L, Sone Y, Silber S. Minimal ovarian stimulation (mini-IVF) for IVF utilizing vitrification and cryopreserved embryo transfer. Reprod Biomed Online. 2010;21(4):485-95. doi:10.1016/j.rbmo.2010.06.033.

11. Gandhi GN, Allahbadia GN, Kagalwala S, et al. IVF Lite-a new strategy for managing poor ovarian responders. IVF Lite. 2014;1:22-8

12. Allahbadia GN. Have we finally written the obituary for conventional IVF? IVF Lite. 2014;1:1-5.

13. Weghofer A, Margreiter M, Bassim S, et al. Minimal stimulation using recombinant follicle-stimulating hormone and a gonadotropin-releasing hormone antagonist in women of advanced age. Fertil Steril. 2004;81(4):1002-6.

14. Kato K, Takehara Y, Segawa T, et al. Minimal ovarian stimulation combined with elective single embryo transfer policy: agespecific results of a large, single-centre, Japanese cohort. Reprod Biol Endocrinol. 2012;10:35. doi:10.1186/1477-7827-10-35. 
15. Check JH, Choe JK, Cohen R. Successful pregnancy following a single fresh embryo transfer in a 45-year-old woman whose early follicular phase serum follicle stimulating hormone was $29 \mathrm{mIU} /$ ml. Clin Exp Obstet Gynecol. 2011;38(4):335-7.

16. Zhu D, Zhang J, Cao S, et al. Vitrified-warmed blastocyst transfer cycles yield higher pregnancy and implantation rates compared with fresh blastocyst transfer cycles-time for a new embryo transfer strategy? Fertil Steril. 2011;95(5):1691-5. doi: 10.1016/j.fertnstert.2011.01.022.

17. Kalampokas T, Sofoudis C, Boutas I, et al. IVF/ICSI frozen replacement cycles; every cycle? Opinion expressed after a systematic review of the literature. Clin Exp Obstet Gynecol. 2015;42(2):173-5.
18. Zhang L, Yan LY, Zhi X, et al. Female fertility: is it safe to "freeze?". Chin Med J. 2015;128(3):390-7. doi:10.4103/03666999.150115.

19. Zhang JJ, Merhi Z, Yang M, et al. Minimal stimulation IVF versus conventional IVF: a randomized controlled trial. Am J Obstet Gynecol. 2015. doi:10.1016/j.ajog.2015.08.009.

20. Bodri D, Kawachiya S, De Brucker M. Cumulative success rates following mild IVF in unselected infertile patients: a 3-year, single-centre cohort study. Reprod Biomed Online. 2014;28(5):572-81. doi:10.1016/j.rbmo.2014.01.002.

21. Gleicher N, Weghofer A, Barad D. Too old for IVF: are we discriminating against older women? J Assist Reprod Genet. 2007;24(12):639-44. doi:10.1016/j.ajog.2015.08.009. 